\title{
IV. Beginn und Ausbau der Spracharbeit im In- und Ausland (1930-1939)
}

Seit 1928 bahnte sich, wie im vorangegangenen Kapitel gezeigt, ein neues Konzept für die Deutsche Akademie an, das auf der Hauptversammlung in Jena im Oktober 1929 schließlich zur Maxime erhoben wurde: Inhaltlich die Sprachwerbung in der Kulturarbeit zum Ausland in den Vordergrund zu stellen, das Engagement für die Auslandsdeutschen hingegen zurückzufahren, und sich geographisch - auch bei den Arbeiten der wissenschaftlichen Abteilung - vor allem auf den Balkan zu konzentrieren. Bereits 1928 bekundete Reichsaußenminister Stresemann seinen Willen, die Deutsche Akademie finanziell zu unterstützen. Sie erhielt 1929 vom Auswärtigen Amt eine einmalige Zahlung von 50000 RM. ${ }^{1} \mathrm{Da}$ man nun im Auswärtigen Amt der Deutschen Akademie wohlwollender als in ihren ersten Jahren gegenüberstand, ja, sie nach der Entdeckung der Spracharbeit und der Ausrichtung auf den Balkan als ein durchaus fördernswertes Instrument auswärtiger Kulturpolitik ansah, zeigte sich am deutlichsten auf der Jahresversammlung im Oktober 1930: Reichsaußenminister Curtius wertete sie durch seine Anwesenheit auf. 1931 erhielt die Deutsche Akademie zum ersten Mal einen festen jährlichen Zuschuß von 8000 RM für die Spracharbeit. Im Jahre 1932 stieg der Zuschuß auf 19000 RM bei einem Gesamtetat der Akademie von 133360 RM. 1933 steuerte das Auswärtige Amt 36500 RM zum Haushalt von 136600 RM bei. In diesem Jahr wurden die Ausgaben für die Sprachwerbung nach jenen für die Verwaltung erstmals zum zweitgrößten Etatposten. ${ }^{2}$ Mitte der dreißiger Jahre, als das Budget der Deutschen Akademie auf etwa 300000 RM anwuchs, belief sich der jährliche Zuschuß der Kulturabteilung auf 65000 RM, der jedoch, wie schon seit 1931, gelegentlich durch Sonderbeihilfen aufgestockt wurde. Dieser Zuschuß war nur für die Spracharbeit im Ausland, vor allem die Lektorengehälter, und für die allgemeine Verwaltung der Akademie gedacht und durfte nicht für die wissenschaftliche Abteilung verwendet werden.

\section{1. „Die Sprache als Bildnerin der Völker“}

Seit 1930 bemühte sich die Deutsche Akademie, auch ein theoretisches Fundament zur Rechtfertigung ihrer vor allem auf Sprachwerbung aufbauenden Auslandsarbeit zu legen. Auf der Hauptversammlung der Deutschen Akademie in Berlin im Oktober 1930 führte Thierfelder aus, daß zwar das Treffen im Vorjahr in Jena den Weg frei gemacht habe für die Schwerpunktverlagerung der Arbeit der praktischen Abteilung auf die Förderung der deutschen Sprache im Ausland.

1 Schlicker, Die Deutsche Akademie, S. $55 \mathrm{f}$.

2 BHStA StK 7390, Aufstellung über den Etat der Deutschen Akademie 1932/33. 
Man könne aber nicht bloß Frankreich oder andere Nationen kopieren, sondern die deutsche Kulturwerbung im Ausland bedürfe einer geistigen Grundlegung. Das Problem Deutschlands beim Kulturexport sei, daß es keine einheitliche nationalen Kultur besitze, die man dem Ausland präsentieren könne. Frankreich verbreite seinen Zivilisationsbegriff, Großbritannien exportiere seinen Lebensstil, die UdSSR und Italien seien zu Repräsentanten einer politischen Ideologie geworden und die USA würden im Ausland mit technologischem Fortschritt gleichgesetzt.

„Wir sprechen zwar gern von deutscher Kultur und jeder einzelne hat eine sehr lebendige Vorstellung von diesem für den Fremden so schillernden Begriff; doch diese Vorstellung ist vorderhand noch ganz persönlich bedingt und nicht in eine Scheidemünze umgeprägt, die von Hand zu Hand gegeben werden kann. Dies aber setzt Kulturpropaganda im westlichen Sinne voraus; sie verlangt Typisches, eine allgemeingültige nationale Form, die überall und zu jeder Zeit eindeutig als Kulturgut dargeboten werden kann. Wir fühlen sofort, daß Deutschland in dieser Beziehung mit anderen Völkern nicht in Wettbewerb treten kann. Wir besitzen keinen nationalen Lebensstil, von dem man sagen kann, er ist für das deutsche Volk typisch." 3

In Ermangelung eines einheitlichen, exportfähigen Lebens- und Kulturstils wurde in den Augen Thierfelders gleichsam die Sprache selbst zum wichtigsten Kulturgut, das es im Ausland zu präsentieren galt. Französisches „savoir vivre“ und die Ideen der französischen Revolution konnte man nach Auffassung der Deutschen Akademie auch ohne französische Sprache an den Ausländer bringen, sie sprachen ebenso für sich wie das britische Gentleman-Ideal, der italienische Faschismus, der sowjetische Kommunismus oder die Errungenschaften der amerikanischen Industrie- und Konsumgesellschaft. Im Falle Deutschlands hingegen sei die Sprache aber Quintessenz deutscher Kultur, das einzige, was dem Ausländer als einendes, für die ganze Nation im wahrsten Sinne des Wortes sprechendes kulturelles Band zu präsentieren war. Denn Sprache sei nicht nur Träger einer bestimmten nationalen Kultur, sondern ihr Ausdruck selbst. Dies war ein keineswegs origineller Gedanke. Er war in Deutschland bereits im 19. Jahrhundert vor allem durch Wilhelm von Humboldts Schrift von 1836 „Über die Verschiedenheit des menschlichen Sprachbaus und ihren Einfluß auf die geistige Entwicklung des Menschengeschlechts" popularisiert worden, ${ }^{4}$ wurde nun aber von der Deutschen Akademie erstmals konsequent in den Dienst der auswärtigen Kulturpolitik gestellt. Im Januarheft 1931 der „Süddeutschen Monatshefte“, das ganz der auswärtigen Kulturpolitik gewidmet war, erklärte Thierfelder folglich unter dem Titel „Geistige Grundlage kultureller Auslandsarbeit" die Sprachwerbung zum Zentrum aller Kulturarbeit im Ausland. Der Ausländer könne die deutsche Sichtweise der Welt nur verstehen, wenn er die deutsche Sprache beherrsche. Der Artikel schloß mit der Feststellung: „Deutsche Kulturarbeit im Ausland ist Werbearbeit für deutsche

3 Bericht über die 5. Hauptversammlung der Deutschen Akademie (16.-18. 10. 1930), in: MdDA, 5 (1930), S. 277-302, hicr S. $291 \mathrm{f}$.

4 Vgl. Andreas Gardt, Sprachnationalismus in Deutschland zwischen 1850 und 1945, in: Nation und Sprache. Die Diskussion ihres Verhältnisses in Geschichte und Gegenwart, hrsg. von Andreas Gardt, Berlin 2000, S. 247-272. 
Sprache“, selbst wenn diese nicht so formvollendet wie die französische oder so leicht zu erlernen sei wie die englische. ${ }^{5}$

Die Deutsche Akademie ging sogar einen Schritt weiter, um auch ein theoretisches Fundament dafür zu legen, daß Kulturarbeit im Ausland vornehmlich im Gewand der Sprachwerbung erfolgen müsse. Sie förderte zusammen mit der „Notgemeinschaft der Deutschen Wissenschaft" ein Buch, das schließlich im Mai 1932 erschien. Es handelte sich um das von Georg Schmidt-Rohr verfaßte Werk „Die Sprache als Bildnerin der Völker“ ${ }^{6}$ Der Autor unterrichtete Germanistik am Lehrerseminar in Frankfurt/Oder. Schmidt-Rohr hatte schon in einer Flugschrift im Jahre 1917 versucht, den Weltkrieg letztlich als Kampf um die deutsche Kultur zu rechtfertigen, welche sich vornehmlich durch die Sprache definiere. ${ }^{7}$ Nun versuchte er, beeinflußt von den in den zwanziger Jahren in der deutschen Sprachwissenschaft an Boden gewinnenden und von Ferdinand de Saussures Strukturalismus beeinflußten, als „Neo-Humboldtianer“ bezeichneten Germanisten um Jost Trier und Leo Weisgerber, den Nachweis zu führen, daß sich in den Gesetzen der jeweiligen Sprache das Denken und Handeln eines Volkes niederschlage. Sprache sei also nicht nur ein Instrument zur Verbreitung von Ideen, sondern ein tendenziell autonomes, beinahe unkontrollierbares Phänomen, das basierend auf seinen jeweiligen spezifischen grammatikalischen, lexikalischen und phonetischen Eigenschaften dem einzelnen wie dem Gesamtvolk bestimmte Eigenschaften zuweise. Schon unmittelbar vor der Veröffentlichung des Buches hatte Thierfelder das Werk als Beweis dafür angekündigt, daß die Tage des "materialistisch-rationalistischen Zeitalters" gezählt seien, welches Sprache nur als mechanisches Hilfsmittel des Gedankenaustausches betrachte. „Haben wir also erkannt, daß sich in unserer deutschen Sprache die höchsten Werte unseres Volkstums am vollkommensten ausdrücken, daß unsere Sprache gleichsam das große geistige Erbe aller unserer Vorgeschlechter wie in einem von unzähligen Strömen gespeisten Sammelbecken einschließt, so wächst daraus unsere heilige Pflicht, seine Ufer zu erweitern." Thierfelder wandte sich zugleich jedoch gegen jede Art von Sprachimperialismus, indem er schrieb: „Ehrfurcht vor der Sprache verbietet uns freilich, andere Völker propagandistisch zu überschwemmen und ihre Sprachquellen zu trüben, Ehrfurcht vor der Sprache schließt die Vergewaltigung Anderssprechender aus. "8 Schmidt-Rohrs Buch wurde von Thierfelder sofort in einer für die "Mitteilungen“ außergewöhnlich langen Besprechung vorgestellt und enthusiastisch gefeiert: "Georg Schmidt-Rohr hat nun nach unserer Überzeugung den entscheidenden Schritt getan. Er spricht selbstbewußt von einer ,kopernikanischen Umkehr' der bisherigen Auffassung vom Wesen der Sprache: nicht nur Spiegel, nicht nur Ausdruck des Volkstums ist sie ihm, er beweist, daß sie die in dem Men-

5 Thierfelder, Geistige Grundlagen kultureller Auslandsarbeit, S. 229.

6 Georg Schmidt-Rohr, Die Sprache als Bildnerin der Völker. Eine Wesens- und Lebenskunde der Volkstümer, Jena 1932.

7 Ders., Unsere Muttersprache als Waffe und Werkzeug des deutschen Gedankens, Jena 1917. Zu Schmidt-Rohr vgl. Gerd Simon, Georg Schmidt-Rohr, in: Internationales Germanistenlexikon 1800-1950, Bd. 3, hrsg. von Christoph König, Berlin 2003, S. 1630-1632.

8 Franz Thierfelder, Societas Latina, Basic English und die unverbesserlichen Deutschen, in: Deutsche Allgemeine Zeitung, 18. 5. 1932. 
schen wirkende gemeinschaftsbildende Kraft ist, die Sprache schafft Volk", schrieb Thierfelder über das Buch, um dann fortzufahren, „[...] und es ist nicht zuviel behauptet, wenn wir es als ,nationales Manifest' bezeichnen, das nicht nur dem deutschen Volke, sondern jeder Nation des 20. Jahrhunderts schlechthin in großen Linien die geistigen Grundlagen ihres Daseins zeichnet".?

Der von Schmidt-Rohr und Thierfelder postulierten „kopernikanischen Wende", mit der sich vortrefflich theoretisch begründen ließ, daß deutsche Kulturpolitik im Ausland vor allem Sprachwerbung sein müsse, war allerdings kein langes Leben beschieden. Schmidt-Rohr hatte sich in seiner Fokussierung auf die Sprache als die jedes Volk prägende Kraft eindeutig gegen die Auffassung gewendet, Rasse und Vererbung bestimme Gestalt, kulturelle Äußerungen und Sprache eines Volkes. ${ }^{10}$ Vielmehr vertrat er die Ansicht, daß jeder Mensch in einer bestimmten Umgebung jede Sprache erlernen könne, unabhängig von seiner Abstammung. Damit konnte potentiell auch jeder Mensch Reichsangehöriger sein, vorausgesetzt, er wuchs im deutschsprachigen Umfeld auf und eignete sich die deutsche Sprache an. ${ }^{11}$ Sein Standpunkt erhielt Thierfelders vollste Zustimmung, denn dieser wandte sich ganz im Einklang mit Jung gegen eine rein rassisch fundierte Definition der Nation, wie sie im völkischen Lager vorherrschte und die von Jung wie Thierfelder als „blutsmaterialistisch“ abgelehnt wurde. ${ }^{12}$ SchmidtRohrs These wurde folglich schon weniger als ein Jahr nach Erscheinen seines Buches zur Zielscheibe der Kritik seitens der neuen Machthaber. Thierfelder suchte den Schaden für die Deutsche Akademie zu begrenzen, indem er auf der Hauptversammlung im Oktober 1933 erklärte, Schmidt-Rohrs Werk sei trotz abweichender Auffassung in der "Rassefrage“ dennoch von „leidenschaftlich nationaler Stimmung getragen"13. Der Autor wie die Akademie traten dennoch einen Teilrückzug an, indem noch 1933 mit Hilfe der Deutschen Akademie eine zweite Auflage des Buches herauskam, die nicht nur in der Einleitung die Machtübernahme Hitlers bejubelte, sondern zumindest im Titel und in den Kapitelüberschriften eine Abschwächung von Schmidt-Rohrs kühner Interpretation anzudeuten schien: Die Sprache war nun nicht mehr „Bildnerin der Völker“, sondern nur noch ein Faktor, der zur „Volkwerdung " beitrug. ${ }^{14}$ Der Autor, der im übrigen im Mai 1933 in die NSDAP eingetreten war, bedauerte zudem in einer der zweiten Auflage seines Buches beigelegten und zugleich in den „Mitteilungen“ abgedruckten Erklärung ${ }^{15}$, daß seine Behandlung der Sprachfrage ihn fälschlicherweise als „liberalen Fürsprecher des Judentums“ habe erscheinen lassen, obwohl er sich zu-

9 Franz Thierfelder, Die Sprache - Ausdruck oder Inbegriff des Volkstums, in: MdDA, 7 (1932), S. 255-258, hier S. 256 f.

10 Schmidt-Rohr, Die Sprache als Bildnerin der Völker, S. 193, 211 f., 224, 289-303.

11 Ebenda, S. 231.

12 Thierfelder, Die Sprache - Ausdruck oder Inbegriff des Volkstums, S. 257f.; Edgar Jung, Die Herrschaft der Minderwertigen, Berlin ${ }^{3} 1930$, S. 121.

13 Bericht über die 7. Hauptversammlung der Deutschen Akademie 13./14. 10. 1933, in: MdDA, 8 (1933), S. 399-427, hier S. 423.

14 Georg Schmitt-Rohr, Muttersprache. Vom Amt der Sprache bei der Volkswerdung, Jena 21933.

15 MdDA, 8 (1933), S. 381. 
gleich in der zweiten Auflage kritisch zur Judenverfolgung äußerte. ${ }^{16}$ SchmidtRohr blieb auch in dieser zweiten Auflage trotz aller Anfechtungen vorerst seiner Grundthese treu, daß die Rasse nicht das entscheidende Merkmal eines Volkes sei, sondern die Sprache, ja er verstärkte sogar im Hauptteil des Buches noch seine Angriffe auf die Rassetheorie. ${ }^{17}$

Schmidt-Rohrs Buch diente im übrigen auch anderen Kräften in der Akademie als Rechtfertigung dafür, der Sprache in der Arbeit der Münchener Organisation verstärkte Aufmerksamkeit zukommen zu lassen. Einer von ihnen war der Vorsitzende der Sektion für Deutsche Literatur, Sprache, Volks- und Altertumskunde, Friedrich Panzer. Er präsidierte zugleich der „Deutschen Gesellschaft für Bildung", wie sich der damalige deutsche Germanistenverband nannte. In seinem Festvortrag auf der Hauptversammlung der Akademie im Oktober 1932 in München forderte er unter Hinweis auf Schmidt-Rohrs Auffassung von der Sprache als einem wesentlichen Element, das ein Volk auszeichne, vermehrte Forschungen der Akademie über die deutschen Sprache und ein größeres Engagement in der Sprachpflege. Dazu solle die Akademie u. a. durch die Errichtung eines „Reichsamtes für deutsche Sprache" beitragen.18 Am 10. und 11. März 1933 verhandelte die Akademie in Berlin mit der Reichsrundfunkgesellschaft, der „Gesellschaft für deutsche Bildung" und dem Deutschen Sprachverein bereits über die Errichtung eines solchen Sprachamtes. Die anvisierte Institution sollte die Behörden im Sprachgebrauch beraten, Auskünfte an die Öffentlichkeit hinsichtlich sprachlicher Fragen erteilen, gegen übermäßigen Gebrauch von Fremdwörtern vorgehen, das Vokabular der deutschen Sprache auf technischem Gebiet planmäßig und sinnvoll erweitern, eine Zentralstelle zur Beratung von Änderungen in der Rechtschreibung und Aussprache werden, der Ausbreitung der deutschen Sprache im Ausland dienen und schließlich eine umfassende Erfassung sprachlicher Phänomene sicherstellen. ${ }^{19}$ Die Deutsche Akademie und die anderen am Treffen beteiligten Institutionen vertagten dann aber eine Entscheidung wegen der seit Januar eingetretenen politischen Umwälzungen, da man erst einmal die Initiativen der neuen Hitler-Regierung abwarten wollte. ${ }^{20}$

Die praktische Arbeit der Deutschen Akademie auf dem Gebiet der Sprachförderung schlug sich ab 1930 in zwei Initiativen nieder, die künftig zu den Pfeilern schlechthin der Auslandstätigkeit der Deutschen Akademie werden sollten: Zum einen bemühte die Münchener Einrichtung sich nun um die Verbesserung der $\mathrm{Me}-$ thodik des Deutschunterrichts im Ausland, zum zweiten gründete die Deutsche Akademie Sprachschulen außerhalb der Reichsgrenzen. Die Spracharbeit entwik-

16 Schmidt-Rohr, Muttersprache, S. 309.

17 Gerd Simon, Wissenschaft und Wende 1933. Zum Verhältnis von Wissenschaft und Politik am Beispiel des Sprachwissenschaftlers Georg Schmidt-Rohr, in: Das Argument, 28 (1986), S. 527-542; Christopher M. Hutton, Linguistics in the Third Reich. Mother-tongue Fascism, Race and the Science of Language, London u.a. 1999, S. $288 \mathrm{ff}$.

18 Abgedruckt in: MdDA, 7 (1932), S. 401-414.

19 Gerd Simon, Sprachpflege im Dritten Reich, in: Sprache im Faschismus, hrsg. von Konrad Ehlich, Frankfurt/M. 1989, S. 58-86, hier S. $61 \mathrm{f}$.

20 BAB R8043/1330, Bericht über die Tätigkeit der Deutschen Akademie 1932/33, Vorlage für die Hauptversammlung im Oktober 1933. 
kelte sich in den dreißiger Jahren im übrigen kontinuierlich, ungeachtet der durch die nationalsozialistische Machtübernahme hervorgerufenen ideologischen Kontroversen über Schmidt-Rohrs Versuch ihrer theoretischen Fundierung.

\section{Die Gründung des Goethe-Instituts}

Im Juli 1930 lud man erstmals 20 Deutschlehrer zu einem von der Deutschen Akademie organisierten und vom Auswärtigen Amt subventionierten Fortbildungskurs nach München ein. Es handelte sich um bulgarische Germanisten, denen Thierfelder nach seiner ersten Balkanreise im Juli 1929 besondere Defizite in der Unterrichtsmethodik attestiert hatte. Die Einladung ausländischer Deutschlehrer zu Sommerkursen nach München wurde seit dem Erfolg des ersten Kurses zum festen Bestandteil der Arbeit der Deutschen Akademie und bis zum Sommer 1944 fortgeführt. Der inhaltliche Schwerpunkt dieses ersten wie der folgenden Kurse lag eindeutig auf Fragen der Methodik des Deutschunterrichts, aber auch landeskundliche und kulturhistorische Themen sowie ein Kultur- und Ausflugsprogramm wurden stets geboten.

Die Kurse basierten auf der Annahme, daß man keinen Einfluß auf die ausländischen Lehrpläne zwecks Ausdehnung des Deutschunterrichts nehmen könne. Statt dessen müsse man das Fach Deutsch zumindest attraktiver gestalten, indem man die ausländischen Deutschlehrer durch Einladungen nach München motivierte und mit modernen didaktischen Methoden vertraut machte. Insbesondere in den noch relativ jungen Staaten des Balkans stellte die Deutsche Akademie Nachholbedarf fest. Diese hätten häufig nicht die Mittel, ihren Deutschlehrern eine ausreichende pädagogische Ausbildung und Fachdidaktik vergleichbar jener zu vermitteln, welche die deutschen Lehramtsanwärter während ihrer Referendarszeit erhielten. ${ }^{21}$ Ein verbesserter Unterricht wiederum sei auch ein Mittel, um dem der deutschen Sprache vorauseilenden Ruf, im Vergleich zum Englischen oder Französischen eine schwierige Sprache zu sein, entgegenzutreten. Da die deutsche auswärtige Kulturpolitik und damit die Deutsche Akademie auch nicht die gleichen Mittel wie die französische oder italienische Konkurrenz verfüge, müsse man eben Quantität durch mehr Nachdruck auf die Qualität des Deutschunterrichts ersetzen, der im Ausland nach Möglichkeit durch die modernsten didaktischen Erkenntnisse vermittelt werde solle. ${ }^{22}$

Um die Fortbildungskurse für ausländische Deutschlehrer in München dauerhaft zu etablieren und eine Stätte zur Erfassung bestehender Methoden und Unterrichtsmittel sowie deren Weiterentwicklung zu schaffen, schlug Thierfelder 1931 die Einrichtung eines entsprechenden Instituts im Rahmen der Deutschen Akademie vor. Dieses sollte zugleich auch die künftigen deutschen Sprachlehrer im Ausland ausbilden. Thierfelder sah es auch als zentrale Auskunftsstelle für alle

21 Erich Drach, Deutsche Sprachbildung im Ausland, in: MdDA, 6 (1931), S. 162-177, hier S. $169 f$.

22 Thierfelder, Deutsche Spracharbeit in Südosteuropa, S. 263. 
Fragen des Deutschunterrichts, die im Ausland auftauchen könnten. Die Erforschung des Deutschunterrichts für Nichtmuttersprachler, heute als „Deutsch als Fremdsprache" $(\mathrm{DaF})$ an vielen in- und ausländischen Universitäten als Studienfach und Forschungsfeld fest etabliert, war ein Bereich, der - im übrigen bis in die sechziger Jahre des zwanzigsten Jahrhunderts hinein - von den germanistischen Instituten der deutschen Universitäten ignoriert worden war. ${ }^{23}$ Thierfelder und der in den dreißiger Jahren eng mit dem Goethe-Institut zusammenarbeitende Berliner Universitätslektor und frühere Schauspieler Erich Drach, eine der Autoritäten der damaligen Zeit in Fragen der Sprecherziehung und Rhetorik, fühlten sich daher als Pioniere in einem bislang von der Germanistik vernachlässigten, ihrer Meinung nach aber kulturpolitisch ungeheuer wichtigen Feld. „Eine Methodik des Deutschen als Fremdsprache gibt es nicht, ebenso gibt es keine Methodik des Erwachsenenunterrichts. Es muß alles neu aufgebaut werden“, hieß es beispielsweise im Protokoll der ersten Versammlung der Sprachlehrer der Deutschen Akademie im Juni 1933.24

Einzig das "Deutsche Institut für Ausländer" an der Friedrich-Wilhelm-Universität in Berlin hatte sich in den zwanziger Jahren der Problematik und den Anforderungen des Deutschunterrichts für Ausländer angenommen. Es war 1922 ursprünglich gegründet worden, um die wachsende Zahl ausländischer Studenten in den Stand zu versetzen, den Veranstaltungen an der Berliner Universität zu folgen. Es begann entsprechende Lehrbücher herauszugeben, vor allem Walter Webers „Deutsch für Ausländer“ (1923) und das 1929 erstmals aufgelegte und bis in die fünfziger Jahre gebräuchliche Werk von Hans Schulz und Wilhelm Sundermeyer, „Deutsche Sprachlehre für Ausländer“. Neben Sprachkursen für Ausländer bot es auch schon seit 1922 Fortbildungskurse für ausländische Deutschlehrer an. Leiter des Berliner Instituts war Professor Karl Remme, der schon 1927 auf die Bedeutung hinwies, ${ }^{25}$ die der Verbreitung der Sprache in der französischen auswärtigen Kulturpolitik beigemessen werde und dadurch möglicherweise Thierfelder beeinflußte.

Thierfelder behauptete in einer Denkschrift ${ }^{26}$ aus dem Jahre 1931, gerade die derzeitige wirtschaftliche und politische Schwäche Deutschlands, die alles Deutsche zunächst als ungefährlich erscheinen lasse, habe im Ausland zu einem vermehrten Interesse an der deutschen Kultur und Sprache geführt, die es zu nutzen gelte. Wenn es gelänge, in München eine feste Einrichtung zur Förderung des Deutschunterrichts für Ausländer zu etablieren, prophezeite er der bayerischen

${ }^{23}$ Vgl. hierzu Dietrich Eggers, Zur Geschichte und zum Selbstverständnis des Faches Deutsch als Fremdsprache aus Sicht der Hochschulen und Universitäten der Bundesrepublik, in: Das Fach Deutsch als Fremdsprache in den deutschsprachigen Ländern, hrsg. von Rolf Ehnert und Hartmut Schröder, Frankfurt/M. u. a. 21994, S. 83-102.

24 PA Bd. R64195, Die Spracharbeit der Deutschen Akademie im Ausland, Protokoll der Tagung vom 28./29. 6. 1933 .

25 Vgl. Roswitha Günther, Das Deutsche Institut für Ausländer an der Universität Berlin in der Zeit von 1922 bis 1945. Ein Beitrag zur Erforschung des Lehrgebietes Deutsch als Fremdsprache, Berlin (Ost) 1988. Vgl. Remme/Esch, Die französische Kulturpropaganda.

$26 \mathrm{BAB} \mathrm{R} 51 / 2$, Vorschläge zur Errichtung eines Instituts zur Fortbildung von ausländischen

Deutschlehrern in München, Denkschrift Thierfelders von 1931. 
Landeshauptstadt nicht nur kostenlose Werbung über die Reichsgrenzen hinaus, sondern auch einen Geldsegen durch den einsetzenden Sprachtourismus. Dieser würde vor allem aus dem zahlungskräftigen Nordamerika kommen, wo der Deutschunterricht sich im vollen Aufschwung befinde und alsbald Hunderte von fortbildungshungrigen und devisenstarken amerikanischen Deutschlehrern nach München führen werde. Mit der Förderung der Gründung des „Instituts zur Fortbildung ausländischer Deutschlehrer" könne die Stadt München zugleich einen herausragenden und dauerhaften Beitrag zum 1932 anstehenden "GoetheJahr“ anläßlich des hundertsten Todestages des größten deutschen Dichters leisten.

Die Prognose einer Invasion der bayerischen Landeshauptstadt durch bildungshungrige US-Deutschlehrer, die tatsächlich nie erfolgte, ist ein Beispiel dafür, wie Thierfelder von ihm selbst postulierte Trends hinsichtlich der Stellung der deutschen Sprache im Ausland nutzte, um mögliche Sponsoren wie die Stadt München für die Arbeit der Akademie zu gewinnen. Da die Deutsche Akademie nach wie vor unter Geldmangel litt, vereinbarte Thierfelder mit der Goethe-Gesellschaft in Frankfurt 1931, daß dem neu zu gründenden Institut als einmaliger Zuschuß ein Drittel der in Bayern im Jahre 1932 eingenommenen Spenden zukommen sollte, die im Jubiläumsjahr des Dichters voraussichtlich ganz besonders kräftig sprudeln würden. Gerechnet wurde mit etwa 4000 RM. Im Gegenzug würde das neugegründete Institut dauerhaft den Namen "Goethe-Institut zur Fortbildung ausländischer Deutschlehrer" tragen. Das Auswärtige Amt war zunächst gegen diese Namensgebung, da sie nur Verwirrung stifte. Doch Thierfelder, der ansonsten in allen Fragen der Sprachförderung aufs engste mit der Wilhelmstraße kooperierte, blieb standhaft: Die Deutsche Akademie benötige unbedingt die Spende der Goethe-Gesellschaft zur Errichtung des Instituts. ${ }^{27}$

Am 22. März 1932, dem Todestag des Namenspatrons, wurde das Institut offiziell gegründet. Im Kuratorium saßen neben dem Präsidenten der Deutschen Akademie Vertreter der Stadt München, des bayerischen Staates und der Münchener Goethe-Gesellschaft. Thierfelder übernahm die Leitung. Seiner ursprünglichen Forderung, daß das Institut auch in einem eigenen Haus untergebracht werden solle, konnte aus finanziellen Gründen allerdings vorerst nicht Rechnung getragen werden. Wegen der Gründung des Goethe-Instituts, für dessen Tätigkeit die Akademie nunmehr erstmals Unterrichtsräume benötigte, zog sie jedoch im Sommer 1932 vom Odeonsplatz, wo das halbe Dutzend Mitarbeiter der Geschäftsstelle seit 1924 gearbeitet hatte, ins Maximilianeum um. Dort mietete man zwei Stockwerke im Südflügel an. Das Maximilianeum blieb bis 1945 Sitz der Akademie.

Das erste Jahr der Tätigkeit des Goethe-Instituts verlief nicht sonderlich spektakulär. Die Kurse waren so konzipiert, daß sie für die Deutsche Akademie einen Gewinn abwerfen sollten. Doch statt der erhofften Kurse für ein halbes Dutzend Nationalitäten kam es wegen der Weltwirtschaftskrise zu einer nur geringen Zahl von Anmeldungen, so daß im ersten Sommer nur ein gemischter Fortbildungs-

27 Der Schriftwechsel zwischen Thierfelder und Auswärtigem Amt zur Namensgebung befindet sich in: PA Bd. R 64194. 
kurs für Italiener, Finnen und Schweden abgehalten werden konnte. Den Unterricht für die ausländischen Germanisten übernahmen außerhalb der rein sprachdidaktischen Module bis in den Krieg hinein zumeist Dozenten der Münchener Universität, darunter der später im Zusammenhang mit der studentischen Widerstandsgruppe „Weiße Rose“ hingerichtete Professor Kurt Huber, mit dem Thierfelder persönlich befreundet war. Erst 1934/35 erhielt das Goethe-Institut zwei eigens angestellte Kräfte namens Magda Gerken und Kurt Derleth, die sich vor allem der Erforschung von Unterrichtsmethoden und der Herstellung von Unterrichtsmaterialien widmeten. Bis 1936 stieg die Zahl der jährlichen Kursteilnehmer auf etwa 300. 1937 erhielt das Goethe-Institut schließlich mit dem sogenannten Ausländerhaus ein eigenes Gebäude in der Galeriestraße 6 am Hofgarten, das mit Kriegsausbruch allerdings aufgegeben wurde. 1937 zählte man schon 768, 1938 schließlich 878 ausländische Kursteilnehmer aus etwa 30 verschiedenen Nationen, wobei Briten, Amerikaner, Italiener und Schweden am stärksten vertreten waren.

Die Ausländerkurse des Goethe-Instituts dienten zugleich der Ausbildung der von der Deutschen Akademie ins Ausland entsandten Lektoren, die hier vor ihrer Ausreise hospitierten. Das Goethe-Institut gründete im Sommer 1933 anläßlich der ersten Tagung der von der Deutschen Akademie ins Ausland entsandten Deutschlehrer, die von nun an jährlich stattfand, eine „Arbeitsgemeinschaft deutscher Lektoren und privater Sprachlehrer im Ausland“ zwecks Erfahrungsaustausch über und Vereinheitlichung des Deutschunterrichts im Ausland. Seit 1935 gab diese Vereinigung das von Thierfelder und Gerken redigierte, zunächst vierseitige, dann achtseitige monatlich erscheinende, kostenlose Blatt mit dem Titel „Deutschunterricht im Ausland“ heraus. Es enthielt praktische Tips zur Gestaltung des Deutschunterrichts, alsbald aber auch Leseempfehlungen für den Unterricht.

Im Juli 1934 eröffnete das "Goethe-Institut", dessen Bibliothek zu diesem Zeitpunkt 3200 Bücher landeskundlicher, literarischer, pädagogischer und sprachwissenschaftlicher Art zählte, eine „pädagogische Schau“ zum deutschen Sprachunterricht im Ausland in vier Räumen des Maximilianeums. Dieses kleine Museum sollte ebenso wie die Fachbibliothek die Ausbildung ausländischer Deutschlehrer unterstützen, die an einem Sommerkurs des Goethe-Instituts teilnahmen. Die Ausstellung befaßte sich mit der Verbreitung der deutschen Sprache im Ausland und den dafür arbeitenden Institutionen. Sie präsentierte ferner Hilfsmittel des Sprachunterrichts, zeigte einen idealtypischen Unterrichtsraum zur Vermittlung der deutschen Sprache, versuchte den Stand der damaligen Pädagogik hinsichtlich des Fremdsprachenunterrichts zu vermitteln und widmete sich der Sprachbildung und Sprecherziehung, angefangen bei den anatomischen Voraussetzungen des Menschen zur Lautbildung.

\section{Die „direkte Methode“}

Die Fortbildungskurse für in- wie ausländische Germanisten am Goethe-Institut seit 1932 zielten vor allem auf die Unterweisung in der seit den zwanziger Jahren in Deutschland im Fremdsprachenunterricht voll durchgesetzten "direkten Me- 
thode" ab. Sie betonte anstelle des überkommenen grammatik- und schriftsprachenlastigen Unterrichts die Vermittlung des gesprochenen Wortes und verzichtete weitgehend auf die Zuhilfenahme der Muttersprache. Die "direkte Methode“ wurde konsequent bei den Grundkursen der Auslandslektorate der Deutschen Akademie angewandt.

Der Fremdsprachenunterricht in Deutschland hatte seit dem frühen 19. Jahrhundert im Zuge der Durchsetzung des neuhumanistischen Bildungsideals an den Schulen und der durch die Befreiungskriege aufgekommenen antifranzösischen Ressentiments die Erlernung der Grammatik und der Schriftsprache nicht nur bei den alten Sprachen, sondern auch bei den modernen Fremdsprachen in den Vordergrund gestellt. Fremdsprachen, so war die unter Pädagogen allgemein verbreitete Auffassung, könne man höchstens durch systematisches Übersetzen anhand eingeübter Regeln erlernen, man würde aber nie in der Lage sein, in ihnen intuitiv $\mathrm{zu}$ denken und zu sprechen. Fremdsprache und Muttersprache wurden stets gegenübergestellt. Ziel des Unterrichts war ohnehin nicht so sehr die mündliche oder schriftliche Beherrschung der fremden Sprache. Statt dessen sollte abstraktes Denken am Beispiel der Grammatik fremder Sprachen geübt werden. Die mündliche Beherrschung einer fremden Sprache wurde höchstens als ein Nebenprodukt, als beinahe abschätzige "Oberkellnerfertigkeit" anvisiert. ${ }^{28}$ Thierfelder selbst bezeichnete sich rückblickend noch in den fünfziger Jahren als „Opfer der indirekten Methode“, die es ihm als junger Mensch verwehrt habe, unbefangen ein Gespräch in Englisch oder Französisch zu führen. ${ }^{29}$ Die Dominanz der "grammatischen Grundorientierung" (Walter Apelt) war im übrigen im 19. Jahrhundert kein rein deutsches Phänomen, sondern dominierte auch in Frankreich und Großbritannien: „Diesen Fremdsprachenunterricht prägte vor allem abstraktes Deduzieren, langatmige Formenanalysen und Übersetzungsexerzitien, rigoroses Vokabelpauken, sowie ständiges Systematisieren, besonders aber fortgesetzter Regelkult." 30

Private Sprachschulen wie die seit den 1880er Jahren sich rasch verbreitenden amerikanischen Berlitz-Schools hingegen hatten mit der "direkten Methode“, die an die Bedürfnisse des immer stärker grenzüberschreitenden Wirtschaftslebens angelehnt war, da sie auf die Kommunikationsfähigkeit in der fremden Sprache abzielte, erstaunliche Ergebnisse erzielt. Es bürgerte sich alsbald allgemein der Begriff „Berlitz-Methode“ für diese Art der Sprachvermittlung ein. Die staatlichen Einrichtungen nicht nur Deutschlands waren also gezwungen nachzuziehen, wollten sie nicht ins Hintertreffen geraten und den Sprachunterricht Privatinstituten überlassen, die mit diesem keine kulturpolitische Missionierungsarbeit verbanden. ${ }^{31}$ In Frankreich mit seinem zentralisierten Schulsystem wurde die „di-

28 Erich Drach, Deutsche Sprecherziehung im Ausland, S. 163.

29 Franz Thierfelder, Die Deutsche Sprache im Ausland. Bd. 1: Der Völkerverkehr als sprachliche Aufgabe, Hamburg 1956, S. 93.

30 Walter Apelt, Vom Lernen und Lehren fremder Sprachen. Grundorientierungen und Methoden in historischer Sicht, Berlin 1991, S. 67 ff. (Zitat S. 79).

31 A. Pfrimmer, Ein halbes Jahrhundert direkter Methode, in: Deutschunterricht im Ausland, 3 (1954/55), S. 60-69. 
rekte Methode" beispielsweise 1902 als verbindlich für den Fremdsprachenunterricht eingeführt. In Deutschland hatte schon 1882 der Pädagoge Wilhelm Viëtor in seiner berühmten Streitschrift „Der Sprachenunterricht muß umkehren“ die Ausrichtung des Fremdsprachenunterrichts auf die Schriftsprache und das Studium der Grammatik kritisiert und die Hinwendung zu einem auf das gesprochene Wort fixierten und anwendungsbezogenen Unterricht gefordert. Dies war eine durchaus zeitgemäße Forderung nach der Reichsgründung, die Deutschland zu einer wirtschaftlichen, politischen und militärischen Großmacht ersten Ranges mit über Mitteleuropa hinausreichenden Interessen gemacht hatte. Es dauerte aber im Reich wegen seines föderalen Bildungssystems und der starken neuhumanistischen, d.h. der Vermittlung anwendungsbezogener Lehrinhalte ablehnend gegenüberstehenden Auffassung von Bildung und Kultur, ${ }^{32}$ noch etwa zwanzig Jahre länger als in Frankreich, bis sich die „direkte Methode“ im Fremdsprachenunterricht durchgesetzt hatte. Die aus dem Reich an die deutschen Auslandsschulen entsandten Lehrer zeigten sich zumeist als unfähig, einen auf aktives Sprachvermögen abstellenden Unterricht etwa im Fach Französisch oder Deutsch (für die nichtdeutschen Schüler) abzuhalten. Dies mußte beispielsweise der Direktor der deutschen Schule in Bukarest und spätere Koordinator des Auslandsschulwesens im Auswärtigen Amt, Franz Schmidt, nach Ankunft an seinem neuen Arbeitsort im Jahre 1900 feststellen. Als der erste Reichskommissar für das Auslandsschulwesen zu einem Besuch in Bukarest eintraf, hatte er, obwohl selbst Neuphilologe, bezeichnenderweise Probleme, sich mit dem rumänischen Erziehungsminister auf Französisch zu unterhalten. ${ }^{33}$ Die Denkschrift des Auswärtigen Amts vom April 1914 über das deutsche Auslandsschulwesen stellte signifikanterweise noch heraus, daß der Englisch- und Französischunterricht an den Auslandsschulen, der den Vorgaben der Gastländer folgte und meist von nichtdeutschen Lehrkräften unterrichtet wurde, im Gegensatz zu jenem im Reich "praktisch orientiert sei“. ${ }^{34}$

Die zwanziger Jahre bildeten in der westlichen Welt sprachgeschichtlich ohnehin in etwa die Scheidelinie zwischen einer etwa 150jährigen Dominanz der Schriftsprache als der normenden Sprache und der nun aufgrund technischer Neuerungen wie Telephon, Radio, Grammophon, Tonfilm und Mikrophon standardsetzenden gesprochenen Sprache. ${ }^{35}$ Der Nationalsozialismus war insofern durchaus auch Ausdruck der neuen Sprachära, als er wesentlich mehr Wert auf das gesprochene Wort, allen voran seines „Rhetorik-Talentes“ und „Führers“ Hitler legte denn auf das gedruckte Wort. Das Radio, nicht die Presse wurde auch im 1933 gegründeten Propagandaministerium als das Medium der Zukunft zur Verbreitung politischer Botschaften gesehen. ${ }^{36}$ Das gesprochene Wort rückte auch

$32 \mathrm{Vgl}$. hierzu Bollenbeck, Bildung und Kultur, S. $144 \mathrm{ff}$.

33 Franz Schmidt, Ein Schulmannsleben in der Zeitwende. Lebenserinnerungen von Geheimrat Professor Dr. Franz Schmidt, Marburg 1961, S. 41.

34 Abgedruckt in: Düwell, Deutschlands auswärtige Kulturpolitik, S. 268-370, hier S. 294 f.

35 Peter von Polenz, Deutsche Sprachgeschichte. Vom Spätmittelalter bis zur Gegenwart, Bd. 3: 19. und 20. Jahrhundert, Berlin u. a. 1999, S. 37-39.

36 Goebbels äußerte sich beispielsweise entsprechend am 25.3.1933 in einer Rede vor den 
bei den Sprachkursen der Deutschen Akademie ins Zentrum des Unterrichts. ${ }^{37}$ Aktives Sprechen statt abstrakter Analyse von Satzbau und Stil wurde nun als wesentliches Mittel, ja geradezu Voraussetzung angesehen, um Ausländern deutsches Denken und Fühlen zu vermitteln. ${ }^{38}$

Der Aufbau des Sprachunterrichts im Ausland der Deutschen Akademie fiel somit just in einen Zeitraum, in dem sich in Deutschland ein Paradigmenwechsel im Fremdsprachenunterricht vollzog. So gesehen waren auch in sprachdidaktischer Hinsicht erst in den zwanziger Jahren die Voraussetzungen vorhanden, Deutsch nicht nur in lebendiger Weise im Ausland zu verbreiten, sondern überhaupt die deutsche Sprache als wesentlichen Träger kulturpolitischer Botschaften anzuerkennen. Erst die sich in den zwanziger Jahren im vollen Maße in Deutschland durchsetzende Erkenntnis, daß eine fremde Sprache sehr wohl intuitiv erlernt werden kann und nicht dazu verdammt war, nur als ein gleichsam totes Studienobjekt fremder Grammatikregeln zu dienen, ebnete den Weg dafür, daß man der Verbreitung der deutschen Sprache im Ausland im Auswärtigen Amt und anderen kulturpolitisch tätigen Institutionen mehr Aufmerksamkeit als zuvor widmete. Die von Thierfelder gefeierte Erkenntnis Schmidt-Rohrs, Sprache sei nicht nur Träger von Gedanken, sondern vielmehr Essenz einer Kultur, war ganz wesentlich durch die neue Wertschätzung befördert worden, welche die gesprochene Sprache im Fremdsprachenunterricht erhalten hatte. Sprachkurse mit Dozenten, welche bis zur Jahrhundertwende in den pädagogischen Seminaren Fremdsprachen noch durch „Pauken“ grammatikalischer Regeln erlernt hatten, wären kaum geeignet gewesen, Erwachsene zum anwendungsbezogenen Lernen der deutschen Sprache zu animieren. Auch vor diesem Hintergrund ist es nicht verwunderlich, daß die Gründer der Deutschen Akademie wie Pfeilschifter, Oncken, Haushofer u. a. in der ersten Hälfte der zwanziger Jahre der Spracharbeit als möglichem Aufgabenfeld so wenig Aufmerksamkeit geschenkt hatten. Denn in Erinnerung an ihren eigenen, grammatikzentrierten, leblosen Fremdsprachenunterricht, den sie im 19. Jahrhundert in den Gymnasien des kaiserlichen Deutschlands genossen hatten, erschien die Perspektive, mit diesen Methoden für die deutsche Sprache und damit die deutsche Kultur im Ausland zu werben, wahrlich nicht sehr vielversprechend, sondern eher abschreckend.

Die „direkte Methode“, die in der Deutschen Akademie und auch noch im ersten Jahrzehnt des wiedergegründeten Goethe-Instituts ab 1951 Anwendung fand, vernachlässigte bewußt die Vermittlung grammatikalischer Grundstrukturen zugunsten eines möglichst umfangreichen Alltagswortschatzes. Die fremde Sprache sollte anfangs ohne Hinterfragen in ihren gegebenen Strukturen hin- und aufgenommen werden unter Einsatz aller Sinnesorgane und ohne zu thematisie-

Rundfunkräten des Reichs. Abgedruckt in Helmut Heiber (Hrsg.), Goebbels-Reden, Bd. 1: 1932-1939, Düsseldorf 1971, S. 82-107, hier S. 91.

$37 \mathrm{Vgl}$. die erste Seite des (nicht paginierten) Juniheftes 1935 von „Deutschunterricht im Ausland“: „Seitdem das gesprochene und gehörte Wort und nicht mehr das geschriebene und gelernte im Mittelpunkt des Deutschunterrichts steht, seitdem ist auch Sprecherziehung in den Dienst des deutschen Sprachunterrichts getreten."

38 Drach, Deutsche Sprecherziehung im Ausland, S. 163. 
ren, warum ein Satz in einer bestimmten Weise konstruiert war oder sich Wörter bestimmter grammatikalischer Formen bedienten.

„Das Ziel des Unterrichts besteht darin, daß die Schüler die deutsche Sprache in einem lebendigen Umgang beherrschen. Eine gesprochene, die wirkliche Sprache soll erlernt werden, nicht eine Lehrbuch-Sprache. Der Schüler soll selbst sprechen, sich eine Sprache produktiv aneignen. Das Buch ist infolgedessen nicht die Hauptsache, sondern nur eine Stütze des Unterrichts. Der Unterricht besteht aus einem fortlaufenden Gespräch zwischen Schüler und Lehrer, zu dem in zweiter Linie die schriftlichen Übungen, das Studium nach Lehrbuch und die häuslichen Aufgaben hinzutreten“,

erläuterte Lektor Wilhelm Ruoff in einem Bericht von 1934 an das Auswärtige Amt die Lehrmethode der Deutschen Akademie. ${ }^{39}$ Die praktischen Vorteile der "direkten Methode“ für die Spracharbeit der Deutschen Akademie im Ausland lagen auf der Hand: Sie gab dem Sprachlernenden frühzeitig Erfolgserlebnisse, da er anders als in der jetzt als überaltet geltenden "Übersetzungsmethode“ bald den Eindruck bekam, er könne sich in der fremden Sprache ausdrücken. Sie war einer national heterogenen Gruppe von Schülern leichter zu vermitteln bzw. konnte von Dozenten unterrichtet werden, die der Sprache des Gastlandes nicht oder kaum mächtig waren, da sie auf Erklärungen des „Warum“ weitgehend verzichtete. Schließlich schien sie auch in ihrer spielerischen Art sinnvoller beim Einsatz für berufstätige Erwachsene zu sein, die abends die Kurse der Deutschen Akademie besuchten und nicht das Gefühl vermittelt bekommen sollten, sie würden erneut die Schulbank drücken und müßten wie zu Schulzeiten Grammatik „pauken“.

Die praktische Abteilung der Deutschen Akademie begab sich auch auf unkonventionelle Pfade, um den ausländischen Germanisten zu helfen, ihre Schwierigkeiten beim Vermitteln der deutschen Sprache zu überwinden. So förderte sie seit 1930 die von dem bayerischen Lehrer Georg Lapper entwickelte Methode des "singenden Lernens“. Lapper experimentierte seit seiner Zeit als Lehrer an deutschen Auslandsschulen in China und Spanien vor dem Ersten Weltkrieg damit, den Schülern das deutsche Alltagsvokabular mit Hilfe von auf Basis von Volksliedmelodien gedichteten, einfachen Gesangstexten zu vermitteln, die stets um ein bestimmtes Thema kreisten. ${ }^{40} \mathrm{Die}$ „Methode Lapper" wurde regelmäßig bei den von der Akademie in den dreißiger Jahren veranstalteten Fortbildungskursen vorgeführt und erlebte auch im wiedergegründeten Goethe-Institut nach dem Zweiten Weltkrieg eine kurze Renaissance. Sie wirkte auf den ersten Blick durchaus vielversprechend: „Herr Lapper wußte seine Methode im Lehrgang so gut an den Mann zu bringen, daß alle begeistert waren - ich auch. Und zwar so sehr, daß ich mich bereit fand, quasi als Beitrag zum Lehrgang eine Einführungslektion für Türkisch auszuarbeiten, nach deren Absolvierung wir uns alle fragten, warum wir

39 PA Bd. R63130, Bericht über die Sprachkurse der Deutschen Akademie in Split, März 1934.

40 Siehe Thierfelders Bericht über die Spracharbeit der praktischen Abteilung, in: Bericht über die 5. Hauptversammlung der Deutschen Akademie (16.-18. 10. 1930), in: MdDA, 5 (1930), S. 277-302, hier S. 291-297. 
uns früher beim Erwerb von Fremdsprachen so sehr abgequält hatten“, erinnerte sich ein Lehrgangsteilnehmer aus dem Jahre 1952.41

\section{4. „Gesprochenes Deutsch“}

Das Bedürfnis, ausländische Deutschlehrer fortzubilden, ging einher mit dem Erfordernis, Hilfsmittel für den Deutschunterricht im Ausland zu schaffen. Die ersten Ergebnisse konnte die Deutsche Akademie schon 1930 präsentieren. Sie brachte ein zweibändiges "Sachwörterbuch der Deutschkunde“ heraus, das als Nachschlagewerk für ausländische Germanisten zu allen Fragen deutscher Kultur und Sprache gedacht war. ${ }^{42}$ Zudem begann sie, eine „Zeitgenössische Schriftsteller" genannte Reihe von Leseheften herauszugeben, die in ansprechender, aber dennoch kostengünstiger Aufmachung auf jeweils 32 Seiten den ausländischen Lesern und insbesondere Sprachschülern bekannte neuere Schriftsteller durch Auszüge aus ihren Werken nahebringen sollten. Als erstes erschienen Hefte, die Thomas Mann, Gerhart Hauptmann, Ricarda Huch, Jacob Wassermann und Hans Carossa gewidmet waren. Ausgaben über Hermann Hesse und ab 1933 über Erwin Guido Kolbenheyer und Hans Grimm folgten. Es hatte sich nämlich erwiesen, daß die Unterrichtslektüre in den meisten Ländern bei den deutschen Klassikern des 19. Jahrhunderts stehengeblieben war. Ferner unterstützte die Akademie die Herausgabe einer Aussprachelehre für Ausländer, die durch vier besprochene Schallplatten ergänzt wurde. ${ }^{43}$

Seit Anfang der dreißiger Jahre arbeitete die Deutsche Akademie an einem eigenen, ganz der "direkten Methode" verpflichteten Lehrbuch der deutschen Sprache für Ausländer. Offenbar hielt man jene des Deutschen Instituts für Ausländer für unzureichend, ${ }^{44}$ zumal sie ja vornehmlich für Ausländer verfaßt waren, die bereits in Deutschland lebten, sich also in einem anderen kommunikativen Umfeld befanden als die Kursteilnehmer in den Auslandslektoraten. Drach, der als Autor auserkoren war, legte das Lehrwerk auf zwei Bände an. Der erste Band für die Anfänger war auf reinen Spracherwerb abgestellt, der zweite für fortgeschrittene Sprachschüler sollte stärker die Landeskunde berücksichtigen. ${ }^{45} \mathrm{Da}$ Drach jedoch bereits im Juli 1935 starb, wurde die Arbeit von Gerken und Wolfhart Klee weitergeführt. Letzterer war eigens hierfür von Thierfelder eingestellt worden, der ihn angeblich mit den Worten, „bei mir brauchen sie nicht für die Partei zu arbeiten“, geködert hatte. ${ }^{46}$ Der erste Entwurf des Lehrbuchs wurde auf der Lektoren-

41 Heinz Griesbach, Am Anfang war fast nichts. Wie es vor fünfzig Jahren begann, in: Murnau - Manila - Minsk, S. 73-75, hier S. 73.

42 Walter Hoffstaetter und Ulrich Peters (Hrsg.), Sachwörterbuch der Deutschkunde, 2 Bände, Leipzig 1930.

43 Erich Drach, Deutsche Aussprachelehre für den Gebrauch im Ausland, Frankfurt/M. 1931.

44 So Drach auf der ersten Tagung der Auslandslektoren im Juni 1933 in München, in: PA Bd. R64195.

45 Ebenda.

46 Wolfhart Klee, Erinnerungen - Werke - Dokumente 1909-1985, München 1985, S. 52. 
tagung im Juli 1936 in München mit den Auslandslektoren diskutiert. Die Autoren arbeiteten die dort gemachten Verbesserungsvorschläge ein und schickten das verbesserte Manuskript nochmals an alle Auslandslektoren zur Stellungnahme. ${ }^{47}$ Im Sommer 1939 schließlich erschien der erste Band unter dem Titel „Gesprochenes Deutsch“. Es war ein für damalige Zeiten modernes, aus der Erfahrung vieler im Ausland unterrichtender Dozenten gespeistes, phonetisch vorbildliches Lehrwerk. Mit seiner Betonung der kommunikativen Kompetenz nahm es einen Ansatz vorweg, der erst in den siebziger Jahren des zwanzigsten Jahrhunderts wieder durchgehend in den Lehrbüchern für $\mathrm{DaF}$ aufgenommen wurde. ${ }^{48} \mathrm{Bis}$ Ende 1940 waren bereits 30000 Exemplare gedruckt, bis Kriegsende erschienen 34 Auflagen. "Gesprochenes Deutsch“ wurde in insgesamt 41 Auflagen bis 1968 verlegt.

Das schließlich vorgelegte Lehrbuch folgte Drachs Bestreben, landeskundliche und damit auch potentiell politische Inhalte im Anfängerunterricht zu vermeiden. Tatsächlich drehten sich die Lektionen um Alltagssituationen wie Reisen, Einkaufen, Sportereignisse etc. Folglich war das Lehrbuch in der zweiten Hälfte des Krieges, als die Deutsche Akademie unter die Kontrolle des Propagandaministeriums geriet, hier nicht sonderlich populär. Ein Zeitzeuge glaubte sich sogar erinnern zu können, das Buch sei deshalb ab 1943 aus dem Verkehr gezogen worden, und dem geplanten zweiten Teil „Lebendiges Deutsch“ sogar von vornherein vom Propagandaministerium die Druckerlaubnis versagt worden. ${ }^{49}$ Hier dürfte allerdings eher der Wunsch, die Deutsche Akademie als eine gänzlich harmlose, ja beinahe anti-nazistische Organisation erscheinen zu lassen und damit die eigene Auslandstätigkeit im Kriege der Nachwelt in möglichst mildem Licht zu präsentieren, die Erinnerung geleitet haben. Vielmehr waren bei einem großen Bombenangriff auf das Leipziger Verlagsviertel Anfang Dezember 1943 u.a. 70000 Exemplare des Lehrwerks "Gesprochenes Deutsch“ verbrannt. „Lebendiges Deutsch“ kam bis Kriegsende nicht mehr zur Auslieferung. Auch dies lag vornehmlich daran, daß der vorgesehene Papiervorrat in Leipzig vernichtet worden war..$^{50} \mathrm{~Pa}$ pierknappheit und nicht das Propagandaministerium waren also für den Lehrbuchmangel ab 1943 verantwortlich. Mitautorin Gerken jedoch erwähnte im Februar 1944 auf der letzten Mittelstellenleitertagung der Deutschen Akademie in München die vielfach gegen das Buch geäußerte Kritik. Sie rechtfertigte den „unpolitischen" Ansatz von "Gesprochenes Deutsch" damit, daß in den Anfängerkursen mit einer Methode, die wie die "direkte“ weitgehend auf die Benutzung der Muttersprache verzichte, Unterrichtsthemen und somit auch die Lektionen des Lehrbuches so angelegt sein müßten, daß der Lernende möglichst viel aus seiner eigenen alltäglichen Erfahrungswelt, u.a. unter Zuhilfenahme der Abbildun-

47 Dies geht aus der überlieferten Korrespondenz der Geschäftsstelle mit den Lektoraten hervor, in: BAB R51/23.

48 Eggers, Zur Geschichte und zum Selbstverständnis des Faches Deutsch als Fremdsprache, S. 87.

49 So der ehemalige Mittelstellenleiter der Deutschen Akademie in Frankreich, Gerhard Funke, am 17. 7.1991 in einem Brief an den Autor. Ähnlich Klee in seinen Memoiren, der fälschlicherweise behauptete, Seyß-Inquart habe das Buch 1943 aus dem Verkehr gezogen. Vgl. Klee, Erinnerungen, S. 53.

50 BAB R51/8, Bericht über den Stand der Deutschen Akademie, 23. 12.1943. 
gen im Buch, ableiten und in die fremde Sprache übertragen könne. Nur dann sei ein schneller Fortschritt der Schüler in der fremden Sprache garantiert. Sie vertröstete die anwesenden Lektoren, vor allem aber wohl den vom Propagandaministerium entsandten Generalsekretär Matthias Schmitz damit, daß der in Vorbereitung befindliche Teil II "Lebendiges Deutsch“ sich mehr der Deutschlandkunde widmen werde, also überragende Persönlichkeiten, Glaubens- und Lebensformen, Feste und ähnliches vorstellen werde. ${ }^{51}$

\section{Der Aufbau des Lektoratsnetzes}

Die zweite Säule der Spracharbeit der Deutschen Akademie war neben der Errichtung des Goethe-Instituts in München der Aufbau von Deutschsprachkursen im Ausland, eine Idee, die offenbar auf eine Anregung des Vorsitzenden des Deutschen Schutzbundes im Jahre 1928 zurückging. Das Auswärtige Amt förderte zwar schon in den zwanziger Jahren offenbar einzelne, im Ausland arbeitende Sprachlehrer ${ }^{52}$, doch gab es, bis sich die Deutsche Akademie der Aufgabe annahm, kein systematisches Programm für die Verbreitung der deutschen Sprache im Ausland mittels Erwachsenkursen außerhalb der Universitäten.

Nach Thierfelders beiden vom Auswärtigen Amt finanzierten sprachpolitischen Erkundungsreisen in den Balkan im Juli 1929 und November 1930 nahm das Vorhaben Gestalt an. Die Eröffnung von Sprachkursen im Ausland wurde dennoch anfangs in München durchaus als Wagnis gesehen, da man auf diesem Gebiet keine Erfahrungen hatte. ${ }^{53}$ Die Akademie verfügte zu diesem Zeitpunkt über einen so kurzen finanziellen Atem - aus Geldmangel fand im Jahre 1931 erstmals keine Jahresversammlung statt -, daß sie diese Einrichtungen bei nur geringer Resonanz nicht lange hätte unterhalten können. So hieß es Ende 1930 in den "Mitteilungen“, die Einrichtung von Sprachkursen in Sofia und Split sei geplant. „Falls sie genügend Beteiligung finden, sollen sie zu einer ständigen Einrichtung werden." 54

Die erste Sprachschule, Lektorat genannt, wurde im Frühjahr 1931 in Split, das Thierfelders erste Station auf seiner Reise im November 1930 gewesen war, eröffnet. Der dortige deutsche Konsul hatte Deutschsprachkurse als großes Desiderat bezeichnet, da es in der dalmatinischen Metropole weder eine Universität noch eine deutsche Auslandsschule gab. ${ }^{55}$ Der erste "Auslandslektor" der Deutschen Akademie und gleichsam Urahn aller heutigen Dozenten des Goethe-Instituts war ein bayerischer Lehrer namens Dr. Sprengel, der allerdings nicht lange in den Diensten der Akademie blieb. Die Bezeichnungen Lektor/Lektorat erinnerte

51 BAB R51/26, Protokoll der Tagung der Mittelstellenleiter vom 11.-16. 2. 1944.

52 So findet in den wenigen erhaltenen Akten der Kulturabteilung zum Thema Sprachförderung beispielsweise 1929 ein vom Auswärtigen Amt geförderter privater Sprachlehrer in Neapel Erwähnung.

53 Franz Thierfelder, 25 Jahre deutsche Sprachpolitik im Ausland, S. 225.

54 Nachrichten aus der praktischen Abteilung, in: MdDA, 5 (1930), S. 377.

55 Thierfelder, 25 Jahre deutsche Sprachpolitik im Ausland, S. 225. 
noch an Thierfelders ursprüngliche Idee von 1928/29, die von der Deutschen Akademie aufzubauende Sprachförderung an den ausländischen Hochschulen zu zentralisieren, die durch die parallele Konstituierung des DAAD obsolet geworden war. Dennoch sprach man bis Kriegsende in der Deutschen Akademie von „Lektoraten“, wenn man die Sprachschulen meinte, und von „Lektoren/Lektorinnen " in Hinblick auf die entsandten Lehrkräfte. Noch 1931 eröffnete ein zweites Lektorat in Sofia, geleitet von einem Assessor namens Fred Kaltmann. 1932 nahmen Lektorate in Saloniki und Sarajewo die Arbeit auf, letzteres geleitet von Joachim Schulz, der 1938 erster Leiter der Abteilung Auslandslektorate der Deutschen Akademie werden sollte, und dessen Frau Dora maßgeblich am Wiederaufbau des Goethe-Instituts ab 1951 beteiligt war. Grundsatz der Deutschen Akademie war in den dreißiger Jahren, möglichst dort Lektorate einzurichten, wo es weder eine deutsche Schule noch eine Hochschule gab, an der Deutsch gelehrt wurde. Man konzentrierte sich zumindest in Europa eher auf die größeren Provinzstädte jener Länder, in denen aus wirtschaftlichen, politischen oder kulturellen Gründen mit einem gewissen Interesse an der deutschen Sprache gerechnet werden konnte, die aber in dieser Hinsicht unterversorgt waren, als auf die Metropolen. Aufgrund der in den dreißiger Jahren trotz beginnender Subventionen des Auswärtigen Amts insgesamt bescheidenen Mittel der Deutschen Akademie war es angebracht, die Lektorate nach Möglichkeit in der Provinz anzusiedeln. In den europäischen Metropolen hätten sie kaum mit den kulturpolitischen Stützpunkten der anderen Nationen konkurrieren können. In Haupt- und Universitätsstädten wirkten zudem schon die Lektoren des DAAD als Repräsentanten deutscher Kultur.

Zur Überraschung der Zentrale in München erwiesen sich die meisten Lektorate als voller Erfolg. In Orten, in denen alsbald deutlich wurde, daß es nicht genügend an der deutschen Sprache Interessierte gab oder andere Institutionen konkurrenzstärker waren, scheute man sich auch nicht, die Lektorate wieder aufzugeben. So schloß schon 1934 das ein Jahr zuvor in Ankara eingerichtete und 1935 das im Vorjahr auf der jugoslawischen Insel Kortschula eröffnete Lektorat. Doch bei der großen Mehrheit der Orte, in denen die Akademie sich seit 1931 engagierte, bestand ein ausreichender Bedarf nach einem qualitativ hochwertigen Deutschunterricht für berufstätige Erwachsene, so daß die Zeichen insgesamt eher auf Expansion standen.

Anfang 1934 verfügte die Deutsche Akademie bereits über 20 Lektorate, davon sechs in Griechenland, vier in Jugoslawien, jeweils zwei in Bulgarien, der Türkei und Indien, und jeweils eines in Italien, Schweden, Brasilien und Irak. Zu diesem Zeitpunkt zählten alle Lektorate zusammen ungefähr 2000 eingeschriebene Hörer. ${ }^{56}$ Ende 1938 waren es schließlich 45 Lektorate mit 57 Dozenten in 15 Ländern, wobei Jugoslawien mit Lektoraten an neun Orten und 18 Dozenten, Griechenland mit 11 Lektoraten und 12 entsandten Lehrkräften und Bulgarien mit acht Lektoraten und acht Lehrkräften eindeutig den Schwerpunkt der Auslandstätig-

56 Einen nützlichen Überblick über Grundsätze und Stand der Spracharbeit der Deutschen Akademie im Ausland Anfang der dreißiger Jahre bietet Wilhelm Ruoff, Lehrgänge der deutschen Sprache im Ausland, in: MdDA, 9 (1934), 229-243. 
keit bildeten. Die Zahl der Hörer war auf etwa 7000 angestiegen, davon etwa 4000 in den genannten drei Ländern.

Die Dislozierung der Lektorate auf dem Balkan entsprach nach wie vor Thierfelders sprachpolitischer Lageeinschätzung von 1929: Die Deutsche Akademie wurde vor allem in strategisch wichtigen Ländern tätig, in denen man eine grundsätzliche Bereitschaft zum Deutschlernen vermutete, gleichzeitig aber ein Defizit in der Unterrichtspraxis der Länder und starke sprachpolitische Aktivität anderer Länder, insbesondere Frankreichs, festgestellt hatte. So war Jugoslawien das Schlüsselland für den Zugang zum gesamten Balkan, in dem sich Frankreich eine sprachpolitisch privilegierte Stellung dadurch geschaffen hatte, daß seit 1924 Französisch an allen höheren Schulen erste Fremdsprache, Deutsch jedoch nur Wahlfach war. Bulgarien hingegen galt als Brücke zur Türkei mit einer sprachpolitisch vorerst offenen Situation. Die relativ starke Präsenz der Deutschen Akademie ab 1933 in Griechenland rührte vor allem daher, daß die griechische Regierung seit Anfang der dreißiger Jahre ihren Schülern untersagte, auf ausländische Schulen zu gehen. Da Deutsch aber, anders als Französisch, in den höheren griechischen Schulen keine obligatorische Fremdsprache war, hoffte man in München dieses Defizit durch die Errichtung von Sprachkursen für Erwachsene auszugleichen. ${ }^{57}$ In Griechenland versuchte die Deutsche Akademie bewußt, in solchen Provinzstädten, die keine französischen Kulturstützpunkte hatten, aktiv zu werden, um so im Lande, sozusagen von der Peripherie her, langsam einen sprachpolitischen Umschwung zugunsten des Deutschen herbeizuführen. ${ }^{58}$ Dies gelang bis 1941 nicht nur in Griechenland, sondern in allen drei Ländern, wobei die Entwicklung stets auf Kosten der französischen Sprache ging. In Bulgarien lernten 1931 nur 20\% der Schüler Deutsch als erste Fremdsprache auf den Gymnasien, 80\% hingegen Französisch. 1938 hatte sich der Vorsprung von Französisch nach Einschätzung der deutschen Botschaft in Sofia auf $55 \%$ zu $45 \%$ verringert. ${ }^{59}$ Thierfelder jubilierte Ende 1937, das kulturpolitische Ziel von 1929, Deutsch als Wahlfach in den bulgarischen Progymnasien zu verankern, sei nunmehr erreicht. ${ }^{60}$ In Jugoslawien erfolgte die nach außen hin sichtbare sprachpolitische Wende im Sommer 1940, als Deutsch wieder zur obligatorischen ersten Fremdsprache in den Schulen avancierte. In Griechenland schließlich entthronte Deutsch Französisch als erste Fremdsprache in den Schulen erst nach der deutschitalienischen Besetzung 1941. Allerdings ist davon auszugehen, daß diese Erfolge nicht so sehr auf das Wirken der Deutschen Akademie in diesen Ländern zurückzuführen war. Der sprachpolitische Umschwung war vielmehr Ausdruck einer generellen engeren wirtschaftlichen und politischen Anlehnung der Balkanstaaten an Deutschland seit Anfang der dreißiger Jahre.

Es war von Anfang an beabsichtigt, die Lektorate nicht als bloße Sprachschulen zu führen, sondern als kleine Kulturinstitute. Dies erschien um so nötiger, als es

$57 \mathrm{Vgl}$. Thierfelder, Deutsch im Unterricht fremder Völker IV, S. 347.

58 Thierfelder, 25 Jahre deutsche Sprachpolitik im Ausland, S. 225.

59 Scholten, Sprachverbreitungspolitik des nationalsozialistischen Deutschlands, S. 354.

60 BAB R8043/1331, Erläuterungen zu dem Entwurf des Haushalts 1938 der Deutschen Akademie, Aufzeichnung Thierfelders vom 17.12. 1937. 
bis in den Zweiten Weltkrieg hinein noch keine deutschen Auslandskulturinstitute im eigentlichen Sinne gab. In einigen europäischen Metropolen wie London, Paris, Rom, Budapest und Madrid nahmen seit Ende der zwanziger Jahre lediglich die Zweigstellen des DAAD diese Funktion mittels kleinerer kultureller Veranstaltungen und vor allem mittels auf Studenten abzielender Sprachkurse in einem gewissen Umfang wahr. ${ }^{61}$ Dies geschah allerdings in Ländern, in denen die Deutsche Akademie vorerst ohnehin nicht aktiv war. Die Lektorate des Münchener Vereins sollten Vorträge von Gastrednern veranstalten, Empfänge geben und deutsche Lesezirkel einrichten. „Die ,Skola' ist eben nicht nur eine Schule, sondern sie wird langsam als deutsches Zentrum bekannt, wo deutsche Vorträge und Zusammenkünfte stattfinden, wo deutsche Bücher und Zeitschriften zu entleihen sind, kurz wo ein eigenes deutsches Leben pulsiert. Wir arbeiten immer mehr daraufhin, den Kursen ihren privaten Charakter zu nehmen und die Institution zu festigen. Wir möchten schließlich etwas wie ein Institut zustandebringen", berichtete beispielsweise der Lektoratsleiter in Split im Frühjahr 1934 an das Auswärtige Amt. ${ }^{62}$

Zielgruppe des Sprachunterrichts waren in der Regel Akademiker, Beamte sowie die lokale Wirtschaftselite, z. T. auch die Oberstufenschüler der örtlichen höheren Schulen. In Jugoslawien gelang es Mitte der dreißiger Jahre auch, Deutschkurse für Offiziere der Armee und in Schweden für Polizeibeamte einzurichten. Beides wurde in München als besonderer Erfolg gewertet, erweckte in Drittländern aber auch Befürchtungen, daß die Lektorate mehr als bloßen Sprachunterricht betrieben. So berichtete die Londoner "Times“ am 23. Januar 1937 unter der Schlagzeile „Undesired Activities“ darüber, daß Naziagenten, getarnt als Vertreter der Deutschen Akademie, für einen nur symbolischen Betrag in Schweden angeblich deutsche Sprache, tatsächlich aber NS-Propaganda verbreiteten. Selbst die schwedische Polizei sei darauf hereingefallen und so für die Zusammenarbeit mit der Gestapo geworben worden. Als Folge dieses Berichts kam es zu einer Hausdurchsuchung und der Beschlagnahmung der Korrespondenz des Lektorates in Gefle durch die schwedische Polizei. Dank der politischen Zurückhaltung des Lektors, die ganz im Einklang mit der Philosophie der Akademie stand, konnte aber nichts Belastendes gefunden werden. „Der Vorfall gibt jedoch Veranlassung, nochmals dringend darauf hinzuweisen, daß jede nach außen sichtbare Tätigkeit der Lektoren unterbleiben und daß im Briefwechsel mit der Hauptstelle abfällige Bemerkungen über die Charaktereigenschaften des Volkes, unter dem sie tätig sind und seine öffentlichen Einrichtungen völlig vermieden werden müssen“, ermahnte Thierfelder dennoch die Außenstellen der Akademie in einem Rundschreiben. ${ }^{63}$

Ganz im Gegensatz zum Tenor des „Times“-Artikels sollten die Lektorate den Eindruck vermeiden, sie seien Billigsprachschulen. Thierfelder hatte von Anfang

61 Vgl. Laitenberger, Akademischer Austausch und auswärtige Kulturpolitik, S. 71 f. und S. 119-134.

62 PA Bd. R63130, Bericht über die Sprachkurse der Deutschen Akademie in Split, März 1934.

63 BAB R51/23, Rundschreiben an die Lektorate, 15. 8. 1937. 
an darauf bestanden, daß die Kurse kostenpflichtig sein müßten, da ein unentgeltlicher Lehrgang nur in den Geruch der Propaganda kommen würde und leicht in seinem erzieherischen Wert unterschätzt werde. ${ }^{64}$ Zudem hätten die Finanzen der Deutschen Akademie auch gar keine kostenlosen oder sehr günstigen Sprachkurse erlaubt. Die Gebühren für die Kurse der Deutschen Akademie sollten sich nach den landesüblichen Tarifen für Privatunterrichtsstunden oder nach denen der Kulturinstitute von Drittstaaten im Gastland richten, diese aber keinesfalls unterschreiten. ${ }^{65}$ So zielten sie allein schon durch ihre Gebühren vornehmlich auf die lokale „Elite“ ab, da - und dies entsprach auch dem Credo des Auswärtigen Amts - Kulturpolitik auf die führenden Schichten eines Landes ausgerichtet war, wobei der Sprachunterricht im Gastland nach Auffassung des Auswärtigen Amts vor allem Vorstufe für ein späteres Studium der Ausländer in Deutschland sein sollte. ${ }^{66}$ Die Praxis sah aber teilweise anders aus, denn die Sprachkurse zogen offenbar nicht nur die höheren Schichten des Gastlandes an: „Es ist wohl angebracht, auf die einfacheren Bildungs- und Berufsschichten einen besonderen Blick zu werfen. Es kommen da junge Leute, Arbeiter, Friseurgehilfen, angehende Kellner, die Deutsch lernen wollen, buchstäblich aber ihre eigene Sprache nicht beherrschen. Die Mühe, die hier aufgewendet werden muß, ist kaum vorstellbar. [...] Man darf die Erfolge deshalb auch nicht bescheiden genug einschätzen. Oft ist es einfach ein aussichtsloser Kampf, weil jede Voraussetzung und Schulung fehlt", hieß es entsprechend im ersten Artikel der "Mitteilungen“, der sich mit den Sprachkursen beschäftigte. ${ }^{67}$

Zusätzlich zum Unterricht sollten die Lektoren das leisten, was man heutzutage "pädagogische Verbindungsarbeit" nennt: die örtlichen Deutschlehrer mit modernen Unterrichtsmaterial und Unterrichtsmethoden vertraut machen und für die vom Goethe-Institut veranstalteten Fortbildungslehrgänge für Deutschlehrer werben. Ferner sollte das Lektorat über den Stand des Deutschunterrichts an Schulen und Universitäten vor Ort, die Ausstattung der Bibliotheken mit deutscher Literatur und Büchern über Deutschland berichten und die kulturpolitischen Aktivitäten anderer Nationen beobachten.68 Diese Berichterstattung war eine wichtige Quelle für die von der Akademie seit 1934 veröffentlichten SüdOst-Berichte, welche die deutsche Öffentlichkeit über das Ringen der europäischen Großmächte um Einfluß auf dem Balkan informieren sollten.

Seit Ende der dreißiger Jahre wurden die Lektorate in jedem Land, in dem es mehrere von ihnen gab, von einer sogenannten Mittelstelle koordiniert. Diese befand sich in der Regel in der größten Stadt bzw. Hauptstadt des Landes und machte nun der Zentrale Vorschläge, wo die Errichtung neuer Lektorate sinnvoll sei. Hierüber wiederum beriet die Deutsche Akademie mit dem Auswärtigen

64 PA Bd. R63806, Schreiben Thierfelders an das Auswärtige Amt, 5. 12. 1929.

65 BAB R51/56, Merkblatt zur Errichtung eines Auslandslektorates der DA (ohne Datum).

66 So die Kulturabteilung in einem Redeentwurf für den Reichsaußenminister vom Juni 1930, abgedruckt in: Düwell, Deutschlands auswärtige Kulturpolitik, S. 379-384, hier S. 381.

67 Ruoff, Lehrgänge der deutschen Sprache im Ausland, S. 236.

68 BAB R51/56, Merkblatt zur Errichtung eines Auslandslektorates (ohne Datum). 
Amt, da dieses die Spracharbeit teilweise finanzierte. Der Mittelstellenleiter nahm in manchen Fällen ein Universitätslektorat wahr und war in einigen Balkanländern, sofern es im entsprechenden Land wie etwa Griechenland keine DAADZweigstelle gab, auch für den akademischen Austausch zuständig. Im Januar 1938 schließlich, als Thierfelder aus der Akademie ausschied, wurde in der Zentrale in München offiziell eine Abteilung „Auslandslektorate“ eingerichtet mit einem eigenen Leiter. Der zuvor auf dem Balkan tätige Lektor Schulz übernahm den Posten. Bis Ende 1937 hatte Thierfelder die Lektorate zusätzlich zu seinen anderen Verpflichtungen als Generalsekretär betreut.

\section{Das Profil der Lektoren}

Die ausreisenden Lektoren sollten nicht nur pädagogische Befähigung und Fachkenntnisse haben - in der Regel entsandte man Studienassessoren, die Deutsch studiert hatten -, sondern auch kulturpolitisches Gespür und Einfühlungsvermögen in die fremde Kultur zeigen. Auch nach der Machtübernahme der Nationalsozialisten legte man in München Wert darauf, daß sich der Auslandslektor zwar als selbstbewußter, dem NS-Regime aufgeschlossen gegenüberstehender Deutscher gab, aber nicht als auftrumpfender, besserwisserischer Germanisierer, um nicht von vornherein seine Kulturmission $\mathrm{zu}$ unterminieren. So wandte sich Thierfelder im Juni 1933 an den neu ernannten nationalsozialistischen bayerischen Ministerpräsidenten Ludwig Siebert mit der Bitte, die bayerische Unterrichtsverwaltung möge der Deutschen Akademie geeignete Kandidaten zur Entsendung als Lektoren ins Ausland zur Kenntnis bringen: „Ausgewählte jüngere Germanisten, die in ihrer nationalen Einstellung erprobt sind und gleichzeitig neben besonderer pädagogischer Befähigung Takt und Gewandtheit bei der Einfühlung in fremde Verhältnisse gezeigt haben." Hervorragende Prüfungsergebnisse und Fachkenntnisse seien weniger wichtig als charakterliche Festigkeit, persönliche Gewandtheit und vielfältige Begabungen und Interessen.69 Der Lektor, so der in Split tätige Ruoff 1934 in den "Mitteilungen der Deutschen Akademie“, müsse Zurückhaltung und Bescheidenheit an den Tag legen, denn er sei Gast. Er müsse aber zugleich mit der Bewußtheit und Entschiedenheit auftreten, die seiner großen Sache entspreche, doch diese Entschiedenheit solle nach innen größer sein als nach außen.70

Auf der ersten Tagung der im Ausland tätigen Sprachlehrer der Deutschen Akademie Ende Juni 1933 in München erklärte Drach, der im kulturpolitischen Amt der NSDAP tätig war, die Sprachlehrer der Deutschen Akademie dürften auf keinen Fall als Propagandisten innerdeutscher Angelegenheiten ins Ausland gehen. Es werde von Berlin sogar ausdrücklich empfohlen, zurückhaltend zu sein. Niemand verlange, daß die Lektoren Mitglieder der NSDAP seien. „Aber wenn sie sich über die heutigen deutschen Verhältnisse äußern, so kann es selbstverständ-

69 BHStA MK 40444, Schreiben Thierfelders an Ministerpräsident Siebert, 6. 6. 1933.

70 Ruoff, Lehrgänge der deutschen Sprache im Ausland, S. 234. 
lich nur im Sinne des nationalen Staates geschehen. Gegen die nationale Regierung zu sprechen, ist undenkbar." Besonders auf eine klare Haltung zur "Judenfrage“ werde in Berlin Wert gelegt. Diese sei in Deutschland viel problematischer als etwa in Großbritannien und den USA gelagert, da z.B. in den Krankenhäusern der deutschen Großstädte bislang bis zu $80 \%$ der Ärzte Juden gewesen seien, und das „Berliner Tageblatt“ zur Zeit der Weimarer Republik grundsätzlich nur jüdische Redakteure aufgenommen habe. Thierfelder als Nichtparteimitglied hingegen erklärte den Eintritt in die NSDAP als empfehlenswerten Schritt für die Lektoren, während aus Rücksichtnahme auf das Ausland die Träger der kulturpolitischen Arbeit in der Zentrale keine Parteigenossen sein könnten. Diese Forderung hinsichtlich der Parteimitgliedschaft der Lektoren war wohl eher taktischer Natur und für das Protokoll gedacht, um das Vertrauen der neuen Machthaber zu gewinnen, als ernst gemeint. Denn es war schon im Frühjahr 1933 zu einer Attacke gegen ihn aus den Reihen des Nationalsozialistischen Deutschen Studentenbundes (NSDStB) gekommen, der behauptet hatte, Thierfelder habe mit Ruoff einen eindeutig marxistisch orientierten Lektor nach Split entsandt.7 ${ }^{71} \mathrm{Da}$ aber das bayerische Kultusministerium sich hinter Thierfelder stellte und Präsident von Müller sich jegliche Einmischungen in die inneren Angelegenheiten der Akademie seitens des NSDStB verbat, konnte der Angriff leicht abgewehrt werden.

Thierfelder, obwohl keineswegs gegen das NS-Regime eingestellt (siehe Kapitel V), wollte die Spracharbeit im Ausland von einer plumpen tagespolitischen oder ideologischen Instrumentalisierung zugunsten des Reiches freihalten. So erklärte er, für sämtliche Lektoren bestehe die Pflicht, die Landessprache zu erlernen, denn sie sollten eine Art Mittler der Kulturen sein und nicht einseitige Propagandisten. ${ }^{72}$ In einem seiner regelmäßigen Rundschreiben an die Auslandslektorate vom September 1936 verwies Thierfelder nicht nur auf den nötigen politischen und menschlichen Takt im Umgang mit anderen Völkern, sondern auch darauf, $\mathrm{da}$ man zwar in erster Linie Deutschland, aber nicht nur ihm allein diene, sondern dem geistigen Austausch zwischen den Völkern insgesamt. „Der nochmalige ernste und unmißverständliche Hinweis des Führers auf dem Parteitag der Ehre in Nürnberg, daß der Nationalsozialismus eine arteigene deutsche Angelegenheit ist, die auf andere Völker gar nicht verpflanzt werden dürfe und könne, wird uns bei jeder Entscheidung bewußt bleiben", fügte er als Mahnung an mögliche parteipolitische Eiferer unter den Lektoren hinzu. ${ }^{73}$ Thierfelder bezog sich hierbei vermutlich auf eine Passage Hitlers in dessen Rede auf der „Kulturtagung“ des Nürnberger Parteitags am 9. September 1936, in welcher der Diktator u.a. folgenden „fundamentalen Grundsatz" verkündet hatte: „Es kann kein Mensch eine innere Beziehung zu einer kulturellen Leistung besitzen, die nicht in dem Wesen seiner Herkunft wurzelt." "74

71 Harvolk, Eichenlaub und Hakenkreuz, S. 28 f.

72 PA Bd. R64195, Die Spracharbeit der Deutschen Akademie im Ausland, Protokoll der Tagung vom 28./29.6. 1933.

73 BAB R51/23, Rundschreiben an die Lektorate vom 15. 9. 1936.

74 Adolf Hitler, Reden des Führers auf dem Parteitag der Ehre 1936, München 61936, S. 30. 
Der ersten Lektorengeneration verlangte die Akademie erhebliches organisatorisches und kaufmännisches Geschick ab. Sie wurde ohne jegliche Vorbereitung oder Hilfestellung anderer deutschen Behörden an ihren Einsatzort im Gastland geschickt, um hier ein neues Lektorat einzurichten. Im Vorfeld hatte sich die Deutsche Akademie an dem anvisierten zukünftigen Standort jeweils einen zuverlässigen Korrespondenzpartner gesucht, der für sie den Bedarf für Deutschkurse eruierte. Wenn dieser zum Schluß gekommen war, daß das Potential für ein Lektorat vorhanden sei, entsandte die Akademie einen Lektor. Dieser mußte sich dann um alles weitere kümmern, von der Anmietung der Räume über ihre Einrichtung bis hin zur Werbung für die Kurse. ${ }^{75}$ Dabei war das Lektorat so zu führen, daß es der finanziell kränkelnden Akademie außer dem Lektorengehalt und der Versendung von Lehrmaterial und Zeitschriften keine Extrakosten verursachen würde. ${ }^{76}$ „Meist an kleineren oder mittleren Plätzen tätig, an denen es keine deutsche Schule und keine größere deutsche Gemeinde gibt, bisweilen der einzige Deutschsprachige am Ort, bescheiden bezahlt und ausgestattet, ist er Studienleiter und Schuldiener, Sprachwerber und Vertreter höherer geistiger und künstlerischer Ansprüche," so umschrieb Thierfelder die Tätigkeit des Lektors. ${ }^{77}$ Das Gehalt betrug für einen ledigen Lektor in den dreißiger Jahren monatlich etwa 170 RM, für Verheiratete mit Kindern gab es etwa $250 \mathrm{RM}$. Er verdiente also wesentlich weniger als ein Facharbeiter im Reich. Allerdings konnten die Lektoren ihr Gehalt dadurch aufbessern, daß ihnen außerdem 20\% der Einnahmen aus den Sprachkursen vor Ort zustanden, was natürlich zugleich ein Anreiz war, möglichst aktiv für die deutsche Sprache Werbung zu betreiben. Die Arbeitsbelastung war in den Anfangsjahren sehr hoch. Ruoff in Split berichtete z.B. im Frühjahr 1934 von 42 Stunden Unterricht, die er wöchentlich gab. ${ }^{78}$ Thierfelder feierte denn auch, ganz den neuen Zeitläuften entsprechend, die erste Generation von Auslandslektoren auf der Jahresversammlung der Deutschen Akademie im Oktober 1933 in martialischen Tönen als „Beispiele soldatischer Pflichterfüllung“, die allen Widrigkeiten trotzen würden. „Hier wächst eine Truppe von Soldaten heran, die ihr Ziel kennt und zu jedem Opfer bereit ist."79

Die Akademie bemühte sich stets, die Lektoren gegenüber der Öffentlichkeit nicht nur als Spezialisten für die Verbreitung der deutschen Sprache herauszustellen, sondern als Kenner ihres Gastlandes. Sie seien daher geeignet, auch für die deutsche Wirtschaft in ihren sprachpolitischen Einsatzgebieten aktiv zu werden

75 BAK B307/24, Niederschrift über die Sitzung des Vorstandes am 10. 12. 1952. In dieser Sitzung schilderte Thierfelder, wie man zu Zeiten der Deutschen Akademie Lektorate errichtet habe und empfahl eine ähnliche Vorgehensweise für die nunmehr anvisierten Auslandsstützpunkte des Goethe-Instituts.

76 BAB R51/56, Merkblatt für die Errichtung eines Auslandslektorates (ohne Datum). Das Merkblatt gipfelt in der Feststellung, daß sich bei Beachtung der Ratschläge ein Lektorat lukrativ führen lasse.

77 Thierfelder, Deutsch als Weltsprache, S. 63.

78 PA Bd. R63130, Bericht über die Sprachkurse der Deutschen Akademie in Split, März 1934.

79 „Deutsche Kulturpolitik im Ausland“, Vortragsmanuskript Thierfelders von 1933, in: BHStA MK 40444. 
oder später als Berater für die entsprechenden Regionen in den deutschen Unternehmen Verwendung zu finden. Da die Akademie bis Kriegsausbruch finanziell vor allem von Spenden aus der Wirtschaft abhing, mußte sie ihrer sprachpolitischen Aktivität das Mäntelchen einer direkten außenwirtschaftlichen Verwertbarkeit umhängen. Auf dem Fahrzeug der Sprache würden alle Güter einer Nation befördert, selbst die wirtschaftlichen, auch wenn die Auslandskulturpolitik nicht in erster Linie der Erhöhung der Exportziffern diene, ließ Thierfelder auf der Hauptversammlung der Akademie 1936 in einem Vortrag mit dem Titel „Die Bedeutung der Sprachpolitik im Auslande für die deutsche Wirtschaft" verlauten. 80

Frauen wurden bis in die Kriegszeit hinein nur in Ausnahmefällen ins Ausland entsandt. Das bereits erwähnte Schreiben Thierfelders an den bayerischen Ministerpräsidenten vom Juni 1933 ging ganz selbstverständlich davon aus, daß nur Männer geeignet zur Ausreise seien. Folglich gab es nur eine Handvoll Frauen unter dem bis Kriegsausbruch auf 62 Lektoren angewachsenen Stamm von Auslandslehrkräften. Sie kamen nur in größere Lektorate, die von einem Mann geleitet wurden. Von den Ehepartnerinnen der Lektoren erwartete die Akademie, daß sie unentgeltlich bei der Verwaltung der Lektorate und bei der Ausrichtung des Kulturprogrammes mithalfen und, sofern sie qualifiziert waren, auch bis zu sechs Stunden unbezahlt unterrichteten. ${ }^{81}$ Dora Schulz beispielsweise, eines der Gründungsmitglieder des "neuen“ Goethe-Instituts ab 1951 und dort bis 1970 Abteilungsleiterin für den Bereich Inlandsinstitute, verdiente sich ihre ersten kulturpolitischen Sporen im Ausland als unentgeltliche Hilfskraft ihres Ehemannes in Jugoslawien.

Ein Anspruch auf Altersversorgung war im Gehalt der Lektoren nicht enthalten. Eine jährliche Heimreise wurde dem Lektor nur bezahlt, wenn er sich bereiterklärte, in den Sommerkursen des Goethe-Instituts in München einige Wochen umsonst zu unterrichten. ${ }^{82}$ Nostalgisch erinnerte sich folglich Thierfelder in den fünfziger Jahren bei der Neugründung des Goethe-Instituts an die Anspruchslosigkeit der Lektoren der Deutschen Akademie. Diese stehe im Gegensatz zur nunmehrigen Generation potentieller Auslandsdozenten, die nicht nur höhere Gehälter, sondern auch eine Altersvorsorge verlangten und damit die Auslandskulturarbeit zu einem im Gegensatz zu den dreißiger Jahren teuren Unterfangen machten. ${ }^{83}$ Dennoch, an Bewerbern für ihre Spracharbeit im Ausland mangelte es der Deutschen Akademie in den dreißiger Jahren angesichts der durch die Weltwirtschaftskrise hervorgerufenen Akademikerarbeitslosigkeit nicht. ${ }^{84}$ Auch als sich die wirtschaftliche Lage in Deutschland durch die zügellose Aufrüstungspolitik der Nationalsozialisten besserte, gab es weiterhin genügend Bewerber. Denn

80 Hauptversammlung 1936, in: MdDA, 11 (1936), S. 565-587, hier S. 583.

81 BAB R51/23, Rundschreiben an die Lektorate vom 15. 10. 1937.

82 Thierfelder, 25 Jahre deutsche Sprachpolitik im Ausland, S. 227.

83 BAK B306/25, Protokoll der kombinierten Sitzung des Vorstandes und des Verwaltungsrates des Goethe-Instituts, 21. 3. 1955.

84 BAB R51/35, Bericht über die Entwicklung und den Stand der Auslandsarbeit der Abteilung Auslandslektorate, Entwurf vom Februar 1943. 
in Zeiten, als es noch keinen Massentourismus gab, der akademische Austausch zwischen den Nationen erst am Anfang stand und die Reichsmark im Ausland nicht hoch im Kurs stand, war ein Auslandsaufenthalt vor allem für Philologen ein noch wesentlich verlockenderes, aber auch schwieriger zu realisierendes Unterfangen als heutzutage. ${ }^{85}$

85 „Für einige Befreundete darunter war, wie ich weiß, der Wunsch Deutschland überhaupt zu verlassen, ein wichtiges Motiv ihrer Bewerbung. Denn für die Generation, denen meine Freunde und ich angehörten, war es so gut wie unmöglich, ohne diplomatische (oder großkapitalistische) Beziehungen während des Dritten Reiches, d.h. vor dem Krieg, ins Ausland zu reisen“. Aus einem Brief vom 8. 3. 1991 von Arthur Henkel, ehemals Lektor der Deutschen Akademie, an den Verfasser. 